bioRxiv preprint doi: https://doi.org/10.1101/546309; this version posted April 28, 2020. The copyright holder for this preprint (which was

\title{
Efficient exact associative structure for sequencing data
}

\author{
Camille Marchet*, Mael Kerbiriou and Antoine Limasset* \\ Univ. Lille, CNRS, UMR 9189 - CRIStAL, F-59000 Lille \\ *camille.marchet@univ-lille.fr and antoine.limasset@univ-lille.fr
}

\begin{abstract}
Motivation: A plethora of methods and applications share the fundamental need to associate information to words for high throughput sequence analysis. Indexing billions of $k$-mers is promptly a scalability problem, as exact associative indexes can be memory expensive. Recent works take advantage of the properties of the $k$-mer sets to leverage this challenge. They exploit the overlaps shared among $k$-mers by using a de Bruijn graph as a compact $k$-mer set to provide lightweight structures.

Results: We present Blight, a static and exact index structure able to associate unique identifiers to indexed $k$-mers and to reject alien $k$-mers that scales to the largest kmer sets with a low memory cost. The proposed index combines an extremely compact representation along with very high throughput. Besides, its construction from the de Bruijn graph sequences is efficient and does not need supplementary memory. The efficient index implementation achieves to index the $k$-mers from the human genome with 8GB within 10 minutes and can scale up to the large axolotl genome with $63 \mathrm{~GB}$ within 76 minutes. Furthermore, while being memory efficient, the index allows above a million queries per second on a single CPU in our experiments, and the use of multiple cores raises its throughput. Finally, we also present how the index can practically represent metagenomic and transcriptomic sequencing data to highlight its wide applicative range.

Availability: The index is implemented as a $\mathrm{C}++$ library, is open source under AGPL3 license, and available at github.com/Malfoy/Blight It is designed as a user-friendly library and comes along with samples code usage.
\end{abstract}

\section{Introduction}

Tremendous, ever-growing amounts of DNA and RNA reads are made available through high-throughput sequencing methods. Single RNA, DNA, or metagenome and metatranscriptome samples can contain up to billion reads each. As an example, the NIH Sequence Read Archive (SRA) 1] gathers peta-bases of sequences. Working on such collections of samples is an essential challenge for associative indexation schemes that label each sequence of interest, e.g. all fixed length words ( $k$-mers), with a unique identifier. This fundamental block is a corner stone for a large spectrum of methods in bioinformatics: genome assembly 2], efficient overlap detection among large sequences 3], quick quantification of transcriptomes [4, sequence search in large sequences collections [5], variant detection [6]; and can be identified as a generic need in large scale sequence analysis. Even after the assembly step, indexing very large genomes (e.g., Pinus taeda [7] with $20 \mathrm{Gbp}$ or Ambystoma mexicanum [8] with 32 Gbp) or metagenomes remain a serious difficulty. In order to deal with these magnitudes, numerous efforts focused on designing data structures that perform the generic task of associating pieces of information to words from studied sequences.

The main difficulty remains to design data structures that can handle billions of distinct $k$-mers so that they can scale up to very large instances such as genomes, genome collections or metagenomics datasets. There are commonly three main strategies. The first one is to index fixed-size words $(k$-mers) from sequences in structures that enable presence/absence queries. This strategy often relies on Bloom filters 9 ] although such data structures can only determine the membership of arbitrary $k$-mers and cannot associate information to them. Recently Bloom filters, or other probabilistic sets, have been used to search sequences in thousands of indexed raw datasets [10, or for assembly [11, 12. A second strategy uses fulltext indexes that can localize words of arbitrary length in the sequence or set of sequences. They commonly rely on FM-indexes 13 . These methods offer extremely memory-efficient indexes but at a high construction cost and a reduced throughput compared to hash-based methods. Finally, some data structures propose general associative indexes. Based on hash tables [6] and/or filters [5], they allow to store (k-mer, value) pairs. This way, $k$-mers can be associated with pieces of information of any nature, for instance, with their original dataset(s) [4, or counts 14. The presented work pertains to this latter category.

Pioneer works based on associative structures such as Cortex 6 illustrate the high hash tables cost that can require more than a dozen of bytes per $k$-mer. Cortex enhances the de Bruijn graph built from the union of different strains, species or samples, by associating their datasets of origin to each $k$-mer of the 
bioRxiv preprint doi: https://doi.org/10.1101/546309; this version posted April 28, 2020. The copyright holder for this preprint (which was not certified by peer review) is the author/funder, who has granted bioRxiv a license to display the preprint in perpetuity. It is made available under aCC-BY-NC-ND 4.0 International license.

graph using a hash table. However, Cortex hash scheme was designed mainly for speed, and cannot scale up to more than a dozen datasets. Such difficulty motivated recent improvements 15, 16, either based on a practical and efficient implementation of a minimal perfect hash function (MPHF) [17 or FM-index.

Indexes build upon MPHFs present fast queries using less than 4 bits per $k$-mer for the MPHF itself. However, MPHFs are functions computing a bijection between a key set and its value set, they do not represent sets. Additional information is necessary to reject keys absent from the indexed set (alien keys). In a previous work, we proposed to add a structure to associate to each $k$-mer a hash-based fingerprint [14, in order to obtain a probabilistic associative index. The structure has some false positives due to hash collisions, which depend on the fingerprint size.

More recently, a method [18 took advantage of the possibility to assemble $k$-mers using compacted de Bruijn graphs [19, 20, in the tool Pufferfish. They store the position of each $k$-mer in the set of assembled sequences and can reject alien $k$-mers by seeking their sequence at the position indicated by the index.

Indexing $k$-mers is closely tangled to the notion of the representation of a $k$-mer set. Recently, spectrumpreserving string sets (SPSS) 21] were defined as an exact representation of a multiset of $k$-mers coming from a set of strings of length $\geq k$. In the literature, SPSS indeed narrow this definition to the representation of a $k$-mer set and forget the $k$-mer multiplicities. According to this definition and as noticed in Pufferfish, de Bruijn graphs are relevant SPSS since they collapse redundancy in their vertices that represent the $k$-mers set. In this work, we will explore different SPSSs for indexing $k$-mers. Moreover, there exist numerous efficient de Bruijn graph representations (succinct data structures such as BOSS [22], efficient representations of de Bruijn graphs vertices such as DBGFM [23] or deGSM [24]). However, they differ from the scope of this work since they are not associative structures, or at least not implemented as such.

In this contribution, we propose a novel, exact associative structure for $k$-mers dubbed BLight, able to scale to large datasets while being extremely memory efficient and fast. Although it uses a MPHF, this structure is deterministic and yields no false positives at the query. It enabled the indexation of the 18 billion of 31-mers from the axolotl genome using $62.4 \mathrm{~GB}$ of $\mathrm{RAM}(\approx 27$ bits per $k$-mer) in 76 minutes. We were able to perform 1.4 millions of queries per second on a single core and more than 16 million queries per second using 12 cores with the constructed index. Contrary to works dedicated to a particular application (colored de Bruijn graphs [25], quantification [4]), we propose a generic associative index that can fit a wide range of different purposes that can be used to associate any kind of information to $k$-mers. To this extent, we implement a library for user-friendly integration to various projects. We demonstrate its performances on different datasets to illustrate its potential applications on various issues.

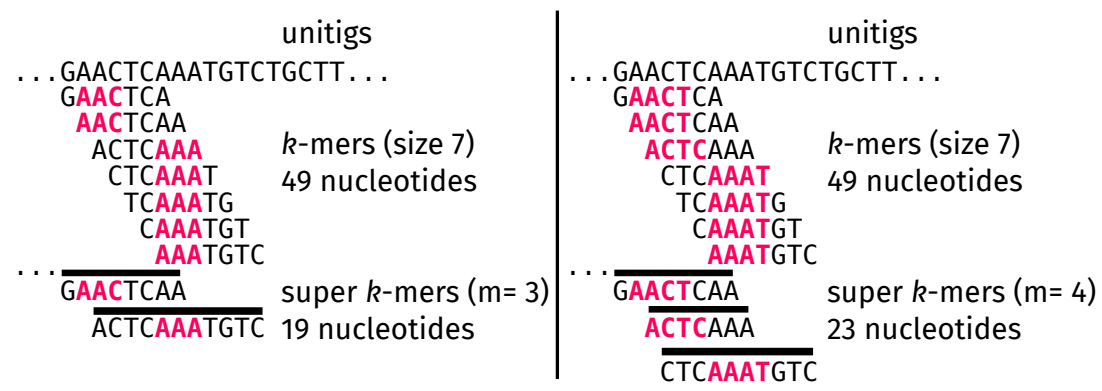

Figure 1: Example of splitting a unitig into super- $k$ mers. The set of 7 -mers uses 49 nucleotides to represent the same information as in the unitig. Left: with minimizers in pink of size 3 . The super- $k$-mers from unitigs use 19 nucleotides. Right: with minimizers of size 4 . Less $k$-mers share the same minimizer, leading to a fragmentation of the super- $k$-mers, and more nucleotides to represent the same information.

\section{Methods}

\subsection{Preliminary definitions}

For simplicity's sake we describe the methods without taking into accounts reverse-complement, in practice a $k$-mer and its reverse-complement will always be considered identical.

Definition 1. de Bruijn graph: The de Bruijn graph is a directed graph $G_{k}=(V, E)$ where $V$ is a set of $k$-mers. For $u, v \in V,(u, v) \in E$ if and only if the suffix of size $k-1$ of $u$ is equal to the prefix of size $k-1$ of $v$. Note that this definition is node-centric: the $k$-mer set is equivalent to the node set and the set of edges can be directly inferred from the nodes set. 
bioRxiv preprint doi: https://doi.org/10.1101/546309; this version posted April 28, 2020. The copyright holder for this preprint (which was not certified by peer review) is the author/funder, who has granted bioRxiv a license to display the preprint in perpetuity. It is made available under aCC-BY-NC-ND 4.0 International license.

Definition 2. Unitig: Given a de Bruijn graph $G_{k}$, a unipath is a maximal-length linear path (sequence of distinct nodes) $s=\left[u_{0}, \ldots, u_{i}, \ldots, u_{n}\right]$ such that

- for each $0 \leq i \leq n-1$, the edge $e=\left(u_{i}, u_{i+1}\right) \in E$;

- in and out-degrees are equal to 1 for each $u_{j}$ such that $1 \leq j \leq n-1$;

- if $n>1$ the out-degree of $u_{0}$ is 1 ;

- if $n>1$ the in-degree of $u_{n}$ is 1 .

Given a unipath $\left[u_{0}, \ldots, u_{i}, \ldots, u_{n}\right]$, a unitig corresponds to the concatenation of nodes in the unipath, such that $u_{0}$ 's string is concatenated with each last character of $u_{i}$ in order, for $1 \leq i \leq n$. Thus, the unitig is a string of length $k+n$. Disconnected $k$-mers of $G_{k}$ are also unitigs.

Definition 3. Compacted de Bruijn graph: The directed graph where nodes are unitigs, and edges are $k-1$-overlaps between two nodes sequences, is called a compacted de Bruijn graph [20]

Definition 4. Minimizer: Minimizers were defined in [26]. The m-minimizer of a sequence $S$ is the smallest substring of size $m$ in $S$, according to some ordering. In BLight, m-minimizers are computed on $k$-mers, hence $m<k$, and the order on $m$-mers is given by a hash function.

\subsection{Outline}

\subsubsection{Inputs/outputs}

For now, we assume that we construct the index from the unitigs of the compacted de Bruijn graph constructed from a $k$-mer set. They can be constructed efficiently with Bcalm2 20] from any FASTA/FASTQ file compressed or not. Later we will see that we can perform the construction from other type of sequences.

For each $k$-mer present in the input graph, the index returns a unique identifier $i \in[1, N]$ with $N$ the total number of $k$-mers, and -1 for any other $k$-mer.

\subsubsection{Index construction}

First, we split the graph into several $k$-mers sets called buckets, according to the minimizers of the $k$-mers (See Figure 1). For a minimizer size $m, 4^{m}$ buckets will be constructed where each bucket will handle all $k$-mers getting a given minimizer. Once filled, we build for each bucket a specific MPHF from its $k$-mer set. Then, for a given bucket and a given $k$-mer, the MPHF returns a unique identifier. We also use this identifier to associate to each $k$-mer its position in its associated bucket. This way, at the query time, we will be able to check if the query $k$-mer is effectively in the index and not an alien $k$-mer. A bucket contain: its $k$-mer sequences encoded in binary, an MPHF that associate to each $k$-mer its position in the sequences and the $k$-mer positions. Each $k$-mer position needs $\mathcal{O}\left(\log _{2}\right.$ (bucket_size $\left.)\right)$ bits to be encoded and the $k$-mer sequences need two bits per nucleotide. We choose to encode both the positions and the $k$-mers using bit-vectors to optimize the required space.

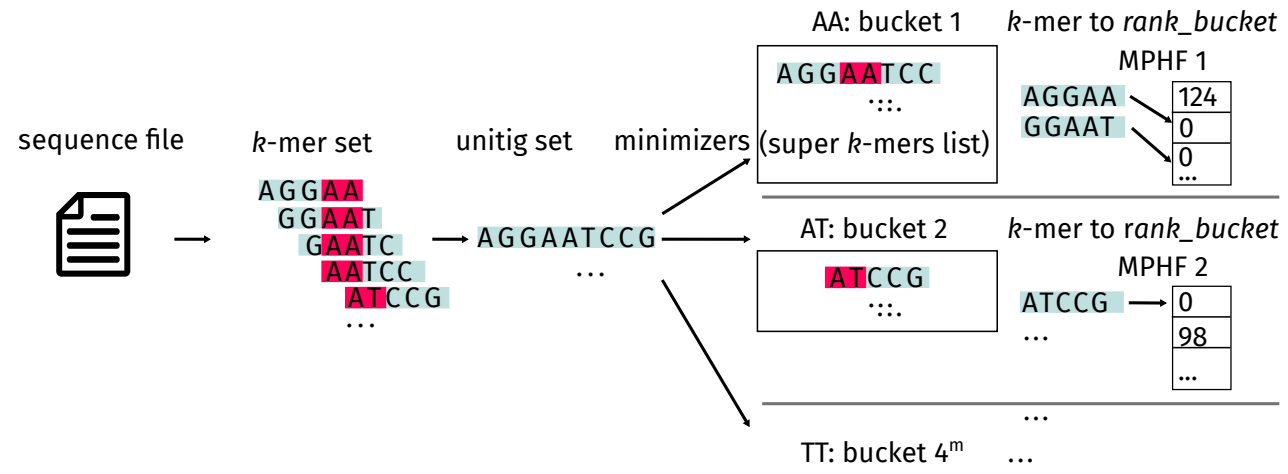

Figure 2: Overview of BLight's method presented in Figure2 We build a compacted de Bruijn graph, i.e., a $k$-mer set, from the initial sequence file. We split unitigs from the graph into super- $k$-mers and put them in buckets, using minimizers (highlighted in pink). When querying, a $k$-mer is looked-up into the relevant bucket using its minimizer. Each bucket is indexed by an MPHF that associates an unique identifier to each $k$-mer. This identifier is also used to associate $k$-mers to their ranks in the bucket. This way $k$-mers can be retrieved in the bucket during the query.

\subsubsection{Query}

The query has two steps (Figure 3 ) : first, we select the relevant bucket according to the query $k$-mer minimizer. Second, we query the MPHF with the $k$-mer. The MPHF can return false positives at query, but no false negatives. For an existing $k$-mer, the MPHF returns its identifier that can be used to get its position in its bucket (Figure 3 (a)). For an alien $k$-mer, the MPHF either returns no information, meaning the $k$-mer is not present, or it falsely returns a position (Figure 3(b)). Then, for any $k$-mer for which the MPHF returned a position $p$, a lookup is performed in the sequences bucket at position $p$. This way, we check that the correct $k$-mer sequence is at the indicated position. If the $k$-mer sequence was not present, or if the MPHF returned no information, we return -1 
bioRxiv preprint doi: https://doi.org/10.1101/546309; this version posted April 28, 2020. The copyright holder for this preprint (which was not certified by peer review) is the author/funder, who has granted bioRxiv a license to display the preprint in perpetuity. It is made available under aCC-BY-NC-ND 4.0 International license.

to indicate this $k$-mer is not in the index. It is thus important to notice that, despite relying on the probabilistic MPHF response, our structure always returns exact answers.

\subsection{Data structure description}

\subsubsection{Super- $k$-mers}

In order to represent $k$-mers from a bucket in a memory-efficient way, we rely on the notion of super- $k$-mers, that appeared in 27. Any sequence can be represented by the list of its $k$-mers, in practice we observe that consecutive $k$-mers often share the same minimizer. Therefore a group of $x$ successive $k$-mers from a sequence sharing the same minimizer can be encoded as a word of length $k+x-1$, called a super- $k$-mer. This technique permit an efficient partition of a multi-set of $k$-mers that use fewer sequences than raw $k$-mers. It is mainly used to compute $k$-mers abundances with a dataset [28].

When first introduced in 28, the super- $k$-mers were computed from reads. In this paper, in order to rely on the partitioning property, we extract super- $k$-mers from unitigs instead of reads (Figure 2). Since we have no duplicate $k$-mers in our unitigs, those super- $k$-mers represent a set of $k$-mers instead of a multi-set.

We use super- $k$-mers in order to store more efficiently $k$-mers in each bucket. We write their sequences and their associated minimizers on the disk in gzipped FASTA format. This way each bucket can be either processed independently to limit the memory usage during the index construction or in parallel.

\subsubsection{Rank structure}

Instead of storing the starting position of a $k$-mer within a bucket, we store in which super- $k$-mer the $k$-mer is present in its bucket. This solution allows using $\log _{2}$ (number_superkmer) instead of $\log _{2}$ (number_nucleotides) bits per $k$-mer in the index, which can represent a difference up to 6 bits per $k$-mer on our experiments. In order to recover the correct super $k$-mers in constant time, we use a compressed rank structure 1 . We construct a bit-array for each bucket, associating a bit to each $k$-mer. A bit is set to 1 if the corresponding super- $k$-mer is the first of its super- $k$-mer. This bit array is compressed and indexed by the rank structure that allows the localization of a given super- $k$-mer in its bucket from the following formula: position_ith_superkmer $=\operatorname{select}(i)+i \times(k-1)$ as each super- $k$-mer is followed by $k-1$ nucleotides that are not indexed $k$-mers. Once the corresponding super- $k$-mer is selected, we check among its $k$-mers if the query $k$-mer can effectively be found. If not, it means that the query $k$-mer is an alien key. This query is constant in practice as the maximal number of $k$-mers within a super- $k$-mer is very unlikely to be above $2 \times k-m$ and is bounded in the implementation.

\subsubsection{Minimizer size and fragmentation}

The number of buckets increases exponentially $\left(4^{m}\right)$ as the minimizer size $m$ increases. As a consequence, buckets contain less $k$-mers (and super- $k$-mers) at higher $m$ values, which means that the bit-vectors used to encode positions in each bucket are also globally reduced (as each $k$-mer position requires $\log _{2}$ (number_superkmer) bits to be encoded). Thus, increasing the size of the minimizers is interesting to encode $k$-mers positions in a more efficient way. However, increasing the number of buckets also means having an additional overhead. Internal information (beginning and the size of buckets), have to be stored for each bucket independently of the input. As this overhead is exponential, it can represent a significant amount of memory when $m$ is above 10. Moreover, another downside of a high $m$ is that overlapping $k$-mers are less likely to share a large minimizer. Therefore, a larger $m$ tends to produce more and smaller super- $k$-mers, and this fragmentation raises the total amount of nucleotide necessary to represent a set of $k$-mer (See Figure 1). An important note is that the graph complexity can also lead to fragmentation as we split unitigs. A very branching graph with very small unitigs will also lead to a high amount of nucleotide to represent a $k$-mer set independently of the minimizer splitting. An interesting fact is that BLight can directly work on any SPSS formatted in FASTA format (i.e., super- $k$-mers would be built not from unitigs, but from another SPSS). It could be especially interesting for large and repetitive genomes that lead to fragmented graphs with a high amount of small unitigs.

\subsubsection{Hierarchical bucketing}

In practice, a uniform partition is hard to obtain. The bucket size distribution is highly unbalanced [23. The smallest minimizers create large buckets, while some buckets can be empty. The largest buckets are notably costly to index because all their $k$-mers will use $\mathcal{O}\left(\log _{2}\right.$ (bucket_size $\left.)\right)$ bits. To tackle this problem, we propose a novel strategy called a hierarchical bucketing scheme. Each bucket will contain smaller $k$-mer sets (sub-buckets) associated with several larger minimizers. By randomly combining smaller sub-buckets, we expect to obtain smaller largest buckets, as it is unlikely to group large sub-buckets. In practice, to fill $4^{m}$ buckets, we compute larger minimizers of length $m+s$, and distribute them to the $4^{m}$ buckets according to a hash function. This way, each bucket contains, on average, $4^{s}$ minimizers of size $m+s$, ensuring a better balance of buckets.

${ }^{1}$ github.com/tlk00/BitMagic 
bioRxiv preprint doi: https://doi.org/10.1101/546309; this version posted April 28, 2020. The copyright holder for this preprint (which was not certified by peer review) is the author/funder, who has granted bioRxiv a license to display the preprint in perpetuity. It is made available under aCC-BY-NC-ND 4.0 International license.

\subsubsection{Sparse index}

We can index super- $k$-mers approximate positions via sub-sampling to reduce the memory impact of the position encoding. The trade-off involves a smaller index for higher query time. More precisely, in order to save $b$ bits per $k$-mer when encoding the positions, one indexes $\tilde{p}=p_{i} \operatorname{div} 2^{b}$ for each $k$-mer $k_{i}$ at position $p_{i}$. From a given position, we have to check at most to $2^{b}$ positions during the query to ensure the $k$-mer exists.

We experimentally show (Table 2 that up to $b=4$, the additional time has a limited impact on the query throughput.
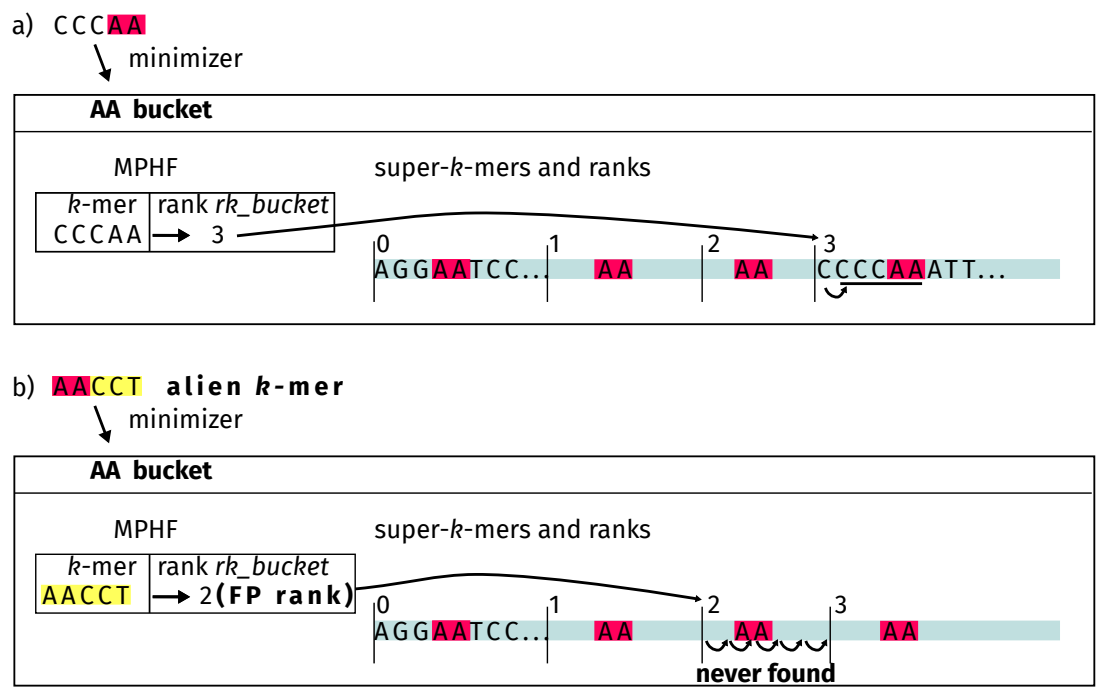

Figure 3: Queries in the index: (a) query of an indexed $k$-mer, (b) query of an alien $k$-mer. Minimizers are represented in pink. Concatenated super- $k$-mers of a bucket are in blue. In (a), after finding the appropriate bucket (box) using the $k$-mer's minimizer, the $k$-mer's super- $k$-mer rank is returned, and the $k$-mer sequence is searched from that position (underlined). In (b), a false positive rank (FP) is returned for the alien $k$-mer, hence it cannot be found in the given super- $k$-mer.

\section{Results}

We benchmark our structure on three different use cases. First, we select large, complex, reference genomes of increasing sizes (starting from the human that represents a moderate reference size, up to the currently largest available at NCBI, i.e., the axolotl Ambystoma mexicanum, with $32 \mathrm{Gbp}$ ) in order to demonstrate how the structure scales to these objects. We use these datasets to demonstrate how the minimizer size impacts the performances of our structure, as well as to illustrate the trade-off obtained when using the sparse version of our index. On this application, we compare the current state-of-the-art approach designed to index such data, Pufferfish.

Second, we demonstrate how our method can handle the indexation of raw reads datasets and show an example of potential applications of such an index. We show that BLight is able to index huge $k$-mer sets from raw NGS datasets, either from metagenomics or transcriptomic samples, up to a huge soil sample containing more than 19 billions distinct $k$-mers. We also used BLight to associate to each $k$-mer its number of occurrences across the datasets to show a straightforward proof of concept application of the library. We selected a dataset from TARA Oceans samples 29, and compare BLight to two lightweight recent $k$-mer abundance indexes: Short Read Connector (SRC) 14 and Squeakr 30 .

Lastly we show that our index can also be used on other SPSS than the unitigs of the compacted de Bruijn graph. We compared the index performances on raw de Bruijn graph and using UST 21] showing a significant gain in performance using this approach.

All experiments were performed on a single cluster node running with Intel(R) Xeon(R) CPU E5-2420@1.90GHz with 192GB of RAM and Ubuntu 16.04.

\subsection{Indexing up to top-largest reference genomes}

Selected genomes To assess the impact of the proposed minimizer partitioning, we built a de Bruijn graph $(k=31)$ from several reference genomes and built the BLight index with different minimizer sizes on their graphs:

- The human reference genome (GRCh38.p12) of 3.2 Gbp, counting 2.5 billions distinct $k$-mers and constructed with Bcalm2 20] using $12 \mathrm{CPU}$ hours and 6.6GB of RAM.

- The latest assembly of Pinus taeda (GCA_000404065.3) of 22 Gbp, counting 10.5 billions distinct $k$-mers and constructed with Bcalm2 using 68 CPU hours and 17.3GB of RAM.

- The latest assembly of Ambystoma mexicanum (GCA_002915635.2) of 32 Gbp, counting 18.3 billions distinct $k$-mers and constructed with Bcalm2 using 107 CPU hours and 44.1GB of RAM. 
bioRxiv preprint doi: https://doi.org/10.1101/546309; this version posted April 28, 2020. The copyright holder for this preprint (which was not certified by peer review) is the author/funder, who has granted bioRxiv a license to display the preprint in perpetuity. It is made available under aCC-BY-NC-ND 4.0 International license.

To compare BLight to Pufferfish, we also included the bacterial genomes graph from Pufferfish paper, constructed from more than 8000 bacterial genomes counting 5.4 billion $k$-mers.

Index construction We constructed the BLight index on the previously mentioned genomes graphs with several minimizer sizes to assess the impact of the minimizer size. We report in Figure 4 the memory necessary to encode the graph super- $k$-mers, to encode the $k$-mers positions, the total amount of memory taken by the index and the actual maximal memory peak during construction. All values are in bits per $k$-mers.

We globally observe that a larger minimizer size leads to a slight augmentation of the memory needed to encode the graph sequences and a decrease of the memory required for position encoding. With uniform bucket filling, we expect the bits required to encode a $k$-mer position to be $\mathcal{O}\left(\log _{2}\left(\frac{\text { genome_size }}{4^{m}}\right)\right)$ resulting in an expected gain of 2 bits per $k$-mer for increasing the minimizer size of one. Interestingly, the observed results are close to this trend in practice on our indexed genomes. We observe that larger minimizers lead globally to smaller indexes because of the reduction of the positions encoding. With a large minimizer size, we can reach the point where the graph itself is more memory expensive than the positions.

However, we also observe the influence of the exponential overhead that becomes prohibitively high after 12 . We also notice that large minimizer sizes suit better huge sets as the high overhead may be too expensive actually reduce the memory usage. In practice, the minimizer size that achieves lowest memory usage is 10 for the human but 11 for the Axolotl.

We summarized the best results obtained with BLight compared to Pufferfish in Table 1 We show that BLight constructs its index using substantially less memory than Pufferfish construction steps but also than Pufferfish index itself. We also show improved construction time, and we want to highlight the fact that unlike Pufferfish, no pre-processing is needed on the graph for the index construction since only the unitig sequences in a FASTA file are needed.

However the comparison is not fair as Pufferfish was designed to be a reference index, able to to associate to each $k$-mer its positions in a reference genome. Therefore Pufferfish needs to keep this information during the construction of the graph and in the index. The memory overhead occasioned by this information is hard to estimate, but we can observe the interest of our partitioning method on the used memory, as a low minimizer size is similar to a strategy without partition.
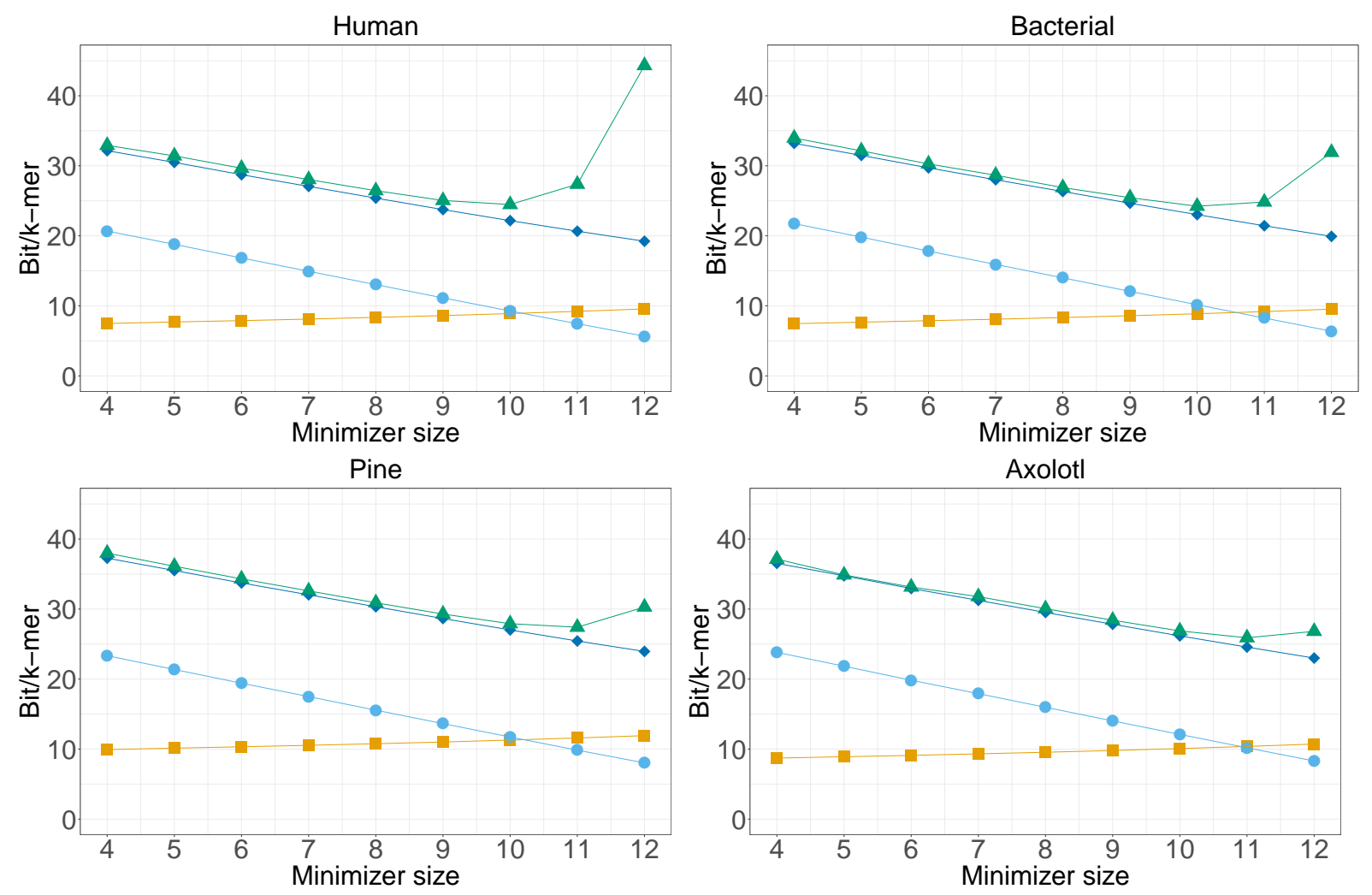

Memory Usage $\|$ Graph • Positions • Index $\bullet$ Peak memory

Figure 4: Detailed memory usage in bits per $k$-mer respectively on the human, bacterial genomes, pine and axolotl graphs during the BLight index construction. 
bioRxiv preprint doi: https://doi.org/10.1101/546309; this version posted April 28, 2020. The copyright holder for this preprint (which was not certified by peer review) is the author/funder, who has granted bioRxiv a license to display the preprint in perpetuity. It is made available under aCC-BY-NC-ND 4.0 International license.

\begin{tabular}{l|c|c|c|c}
\hline Genome & Time (min) & Index Size (GB) & Memory usage (GB) & Bits $/ k$-mer \\
\hline Pufferfish Human & 69 & 17 & 27.4 & 55.59 \\
BLight Human & 10 & 6.62 & 7.4 & 22.3 \\
\hline Pufferfish Bacterial genomes & 187 & 40 & 75 & 114.8 \\
BLight Bacterial genomes & 21 & 15.73 & 16 & 24.23 \\
\hline BLight Pine & 39 & 33.84 & 35.7 & 27.41 \\
\hline BLight Axolotl & 76 & 53.62 & 62.4 & 26.88 \\
\hline
\end{tabular}

Table 1: Comparison of the maximal memory usage in GB and the Wall-clock time in minutes used during construction with BLight and Pufferfish from the de Bruijn graph. We were unable to construct Pufferfish's index for the pine and the axolotl genomes due to memory limitation.

Query time and impact of sub-sampling factor We compared our throughput according to Pufferfish in Table 2. We observe that our query is slightly faster than Pufferfish's on a single CPU. However, using multiple threads significantly raise the query throughput. Either way, both indexes propose a very high throughput of the order of the million queries per second. BLight reaches 1.4 million $k$-mer queries per second on a single core and 16.1 million $k$-mer queries per second using 12 cores. As mentioned before, using a sub-sampling factor enables to control the memory usage at the expense of the query performances. However, we observe that below $b=4$, the impact on the query time is low.

\begin{tabular}{l|c|c||c|c||c|c}
\hline$b$ & $\begin{array}{c}\text { Position encoding } \\
\text { bit } / k \text {-mer }\end{array}$ & $\begin{array}{c}\text { Index Size } \\
\text { GB }\end{array}$ & $\begin{array}{c}\text { Reference (1T) } \\
\text { seconds }\end{array}$ & $\begin{array}{c}\text { Reference (16T) } \\
\text { seconds }\end{array}$ & $\begin{array}{c}\text { Real reads (1T) } \\
\text { seconds }\end{array}$ & $\begin{array}{c}\text { Real reads (16T) } \\
\text { seconds }\end{array}$ \\
\hline 0 & 9.27 & 6.62 & 1,748 & 155 & 1,577 & 122 \\
1 & 8.27 & 6.32 & 1,782 & 159 & 1,728 & 129 \\
2 & 7.27 & 6.02 & 1,931 & 166 & 1,872 & 141 \\
3 & 6.27 & 5.72 & 2,212 & 181 & 2,184 & 165 \\
4 & 5.28 & 5.43 & 2,662 & 210 & 2,813 & 213 \\
5 & 4.28 & 5.13 & 3,484 & 267 & 4,067 & 306 \\
6 & 3.29 & 4.84 & 5,167 & 376 & 6,379 & 486 \\
7 & 2.37 & 4.56 & 8,127 & 592 & 10,760 & 829 \\
8 & 1.65 & 4.35 & 13,137 & 959 & 18,755 & 1,462 \\
\hline Pufferfish & NA & 17 & 1.799 & 1.799 & 1,260 & 1,260 \\
\hline
\end{tabular}

Table 2: Influence of the sub-sampling parameter on the query time. We report the amount of memory used by the positions divided by the number of $k$-mers, the time required to query the real dataset SRR5833294 with one thread, the time required to query the real dataset with 12 threads, the time required to query all the $k$-mers of the reference graph and the time required to query this graph using 12 threads.

BLight as a de Bruijn graph representation BLight can be used as a high-throughput de Bruijn graph representation but is not optimal in comparison to space-efficient graph structures. For each species, we computed the theoretical bound indicated by Conway \& Bromage (CB) 31] for $k=31$ in bits per $k$-mer: human 32.2 , pine 30.15 , axolotl 29.35 and bacterial genomes set 31.11. Contrary to our representation and Pufferfish's, CB does not take into account the possibility to combine the information of multiple adjacent $k$-mer. This fact explains why we can use less space than CB. However, our structure and Pufferfish's use $\mathcal{O}(\log$ (genome_size $))$ bits per $k$-mer, thus could be higher than CB on larger genomes. Other FM-index based methods such as DBGFM maintain compressed representations of the de Bruijn graph, thus can be more space-saving than ours. The proposed methods provide a different trade-off as we expect a faster query time in practice.

\subsection{Indexing $k$-mer from raw reads}

Indexing metagenomic datasets In this section, we assess the ability of the BLight structure to index complex metagenomic datasets. The following experiment is contrasting with the bacterial genome collection used in Figure 4. In the previous experiment, the de Bruijn graph is constructed from reference genomes, while here we use raw datasets, yielding a very different graph: it may contain many sequencing errors and are highly fragmented because of lack of coverage, sequencing errors, and similar genomes present [32. We first chose to index all non-unique $k$-mers of a TARA sample ${ }^{2}$ containing five datasets ERR1712199, ERR1711907, ERR599280, ERR562434, and ERR1718455. BLight was able to build an index from the graph of 7,540,111,917 distinct $k$-mers constructed from Bcalm2. The construction from unitigs lasted less than an hour and used 24.7 GB, which represents 27.1 bits per $k$-mer. This memory usage is higher than the bacterial genomes despite having a comparable amount of $k$-mers. This surplus is due to the expected graph fragmentation of a raw metagenomic sample that raise the amount of nucleotide needed to encode the graph (see Minimizer size and fragmentation section).

2 https://www.ebi.ac.uk/ena/data/view/SAMEA2620556 
bioRxiv preprint doi: https://doi.org/10.1101/546309; this version posted April 28, 2020. The copyright holder for this preprint (which was not certified by peer review) is the author/funder, who has granted bioRxiv a license to display the preprint in perpetuity. It is made available under aCC-BY-NC-ND 4.0 International license.

We also indexed all non-unique $k$-mers of a massive soil sequencing from DOE/JGI Great Prairie Soil Metagenome Grand Challeng $\oint^{3}$ counting 19,289,529,788 distinct $k$-mers. The construction lasted less a day and used 63.8GB, which represents 26.5 bits per $k$-mer.

Indexing transcriptomic datasets In this section, we assess the ability of the BLight structure to index complex transcriptomic datasets. As most transcriptomic sequencing experiments contain a "low" amount of distinct $k$-mers we choose to index all distinct $k$-mers from the 2,585 datasets used for the Sequence Bloom Tree benchmark [10] using the specific filtering method. We build the union de Bruijn graph from all the files using Bcalm2 with $k=31$ and $k=21$. The graphs have 4,425,877,751 distinct 31-mers and 3,903,590,501 distinct 21-mers respectively. Both indexes were built within 30 minutes and used 16.83 GB (30.4 bits per 31-mers) and 16.31 GB (33.4 bits per 21-mers), respectively.

\begin{tabular}{l|c|c}
\hline Tool & Peak memory (GB) & Time (hh:mm) \\
\hline Squeakr exact & 185.4 & $15: 11$ \\
SRC counter exact & 44.20 & $\mathbf{0 5 : 1 2}$ \\
BLight count & $\mathbf{2 0 . 8}$ & $19: 49$ \\
\hline
\end{tabular}

Table 3: Performance comparison of an abundance index construction on the ERR599280 metagenomic dataset.

\subsection{Application example: storing $k$-mer counts}

In this experiment, we provide a proof of concept index, able to associate to each $k$-mer its abundance within a dataset. We compare a simple usage of BLight through a $k$-mer counting snippet, with two methods from state-ofthe-art that allow large scale $k$-mer to abundance association. Squeakr 33 is a $k$-mer counter based on a quotienting hashing technique, and Short Read Connector counter (SRC) 14 is based on a MPHF. We report the performances of the three tools on a large marine metagenomic dataset from TARA used the previous metagenomic experiment (ERR599280), counting 37 billion bases and 189 million reads. All tools are used in exact mode and presented in Table 3. If the memory usage of BLight is very reduced in comparison to the other methods, the index construction is way slower than SRC or Squeakr. This slow performance can be explained by the fact that the proposed snippet has to perform a query on each $k$-mer of the dataset to re-count the $k$-mer abundance. A real implementation should parse a $k$-mer counting result in order to initialize the $k$-mer abundance as SRC. Moreover, SRC or Squeakr inexact modes are expected to be more space-efficient but will yield false positives and inexact results.

\subsection{Benefit of Spectrum-preserving string sets}

In BLight, our first choice was to represent $k$-mer sets in an exact and partitioned way using super- $k$-mers from unitigs. But BLight can construct its index from any Spectrum-preserving string sets without duplicates. We recall that in this work, we are interested in SPSS that represent a set of $k$-mers and will refer to them, and will not take into account multi-sets. Unitigs are one SPSS, super- $k$-mers of unitigs are another 34 . Two other equivalent SPSSs schemes, UST 21] and simplitigs [35, longer than unitigs, were recently independently proposed. The motivation of those two developments is that unitigs are not the most concise representation of a set of $k$-mer, as some compactions could be made in the de Bruijn graph without altering the $k$-mer set they represent.

We used UST to further compact the human and pine unitigs of Figure 1 and compared the amount of memory used on the unitigs versus UST SPSS and report the result in Table 4 We observe improvements in the memory required to encode both the graph sequences and the $k$-mer positions resulting in significantly smaller indexes (from 30 to $26 \mathrm{~GB}$ on the Pine genome with a minimizer size of 12). This show the benefit of using SPSS to represent and index $k$-mer set more concisely and how BLight can benefit from those approaches.

\begin{tabular}{ll|l|l|l}
\hline File & $\begin{array}{l}\text { Minimizer } \\
\text { size }\end{array}$ & $\begin{array}{l}\text { Graph } \\
\text { bits per } k \text {-mer }\end{array}$ & $\begin{array}{l}\text { Position } \\
\text { bits per } k \text {-mer }\end{array}$ & $\begin{array}{l}\text { Total } \\
\text { bits per } k \text {-mer }\end{array}$ \\
\hline Human & 8 & 8.1 & 13.9 & 26 \\
Human UST & 8 & 7.8 & 12.9 & 24.7 \\
\hline Human & 10 & 8.6 & 10.1 & 22.7 \\
Human UST & 10 & 8.3 & 9.1 & 21.5 \\
\hline Pine & 8 & 10.5 & 16.4 & 31 \\
Pine UST & 8 & 8.6 & 15.2 & 27.8 \\
\hline Pine & 10 & 11 & 12.6 & 27.6 \\
Pine UST & 10 & 9.2 & 11.4 & 24.5 \\
\hline Pine & 12 & 11.6 & 8.9 & 24.5 \\
Pine UST & 12 & 9.8 & 7.7 & 21.5 \\
\hline
\end{tabular}

Table 4: Space usage of BLight indexes on raw unitigs compared to unitigs compacted with UST.

3 www.mg-rast .org/mgmain.html?mgpage=project\&project=mgp6377 
bioRxiv preprint doi: https://doi.org/10.1101/546309; this version posted April 28, 2020. The copyright holder for this preprint (which was

\section{Conclusion and future work}

In this work, we propose BLight, a low-cost, high-throughput, and exact associative structure for indexing $k$-mers, relying on de Bruijn graphs or other efficient SPSS. Based on efficient hashing techniques and light memory structure, we believe that the proposed index has a very interesting time/memory compromise, being able to perform millions of queries per second and using less than 32 bits per $k$-mer on our large-scale experiments. In comparison, we show that the closest method, Pufferfish, reaches a different compromise. While not benefiting from the gain in space due to partitioning, it optimize the storage of the $k$-mers positions within a reference genome, making Pufferfish more suitable for applications such as alignment on a reference. Conversely BLight is designed with a more generic application range in mind. We demonstrate that BLight can index the largest available genomes to date using a reasonable amount of memory while outperforming state of the art methods in both construction time and memory. We also showed it could be relevant and efficient on raw transcriptomic and metagenomic sequencing data.

Being able to work on massive datasets is often a challenge in sequence analysis. By providing an efficient structure able to run on medium clusters or laptops, we hope to democratize and lower the inherent cost of this type of analysis. We thus believe that a vast number of methods could rely on and benefit from this structure due to its broad application spectrum. To that extent, we implemented a user-friendly library along with different snippets to allow our method to be usable in practical cases. The challenge of indexing colored de Bruijn graphs 36 (or more generally to answer large sequence search problems as defined in 10]) have caught the interest of a community and could be a direct application of this work. As an example, BLight is successfully integrated as an indexing structure in REINDEER [34, a $k$-mer data structure that enables the quantification of query sequences in thousands of raw read samples.

The main caveat of the proposed structure is that the index cannot include new sequences after its construction. The static aspect allows the index to be extremely memory-efficient, which would be hard to achieve with a dynamic structure. A possible continuation of this work would be a dynamic structure that follow the main idea of BLight, using multiple dynamic indexes partitioned by minimizers. This partitioning should improve the data locality and, therefore, the performances of such a structure. Another way to pursue this work would be to propose a pseudo-dynamic structure using a static and a dynamic structure for the last elements inserted. At some point, in a way to guarantee a constant average insertion time, a complete rebuild could be performed to construct a static index on all elements. More generally, a vast amount of improvements could be brought to the proposed backbone structure. Specific minimizer schemes could be designed to obtain the smallest possible buckets, supplementary structures to sort and rank the super- $k$-mers could allow faster query or reduced position encoding. Such optimizations could improve the global resource usage of the index or provide different time/memory trade-offs.

\section{Acknowledgements}

We would like to thank Rob Patro, Fatemeh Almodaresi, Tatiana Rocher, and Rayan Chikhi for their support and interesting discussions on this project. This work was supported by the ANR Transipedia (ANR-18-CE45-0020).

\section{References}

[1] Rasko Leinonen, Hideaki Sugawara, Martin Shumway, and International Nucleotide Sequence Database Collaboration. The sequence read archive. Nucleic acids research, 39(suppl_1):D19-D21, 2010.

[2] Anton Bankevich, Sergey Nurk, Dmitry Antipov, Alexey A Gurevich, Mikhail Dvorkin, Alexander S Kulikov, Valery M Lesin, Sergey I Nikolenko, Son Pham, Andrey D Prjibelski, et al. Spades: a new genome assembly algorithm and its applications to single-cell sequencing. Journal of computational biology, 19(5):455-477, 2012.

[3] Heng Li. Minimap and miniasm: fast mapping and de novo assembly for noisy long sequences. Bioinformatics, 32(14):2103-2110, 2016.

[4] Nicolas L Bray, Harold Pimentel, Páll Melsted, and Lior Pachter. Near-optimal probabilistic rna-seq quantification. Nature biotechnology, 34(5):525, 2016.

[5] Michael Ferdman, Rob Johnson, and Rob Patro. Mantis: A fast, small, and exact large-scale sequence-search index. In Research in Computational Molecular Biology: 22nd Annual International Conference, RECOMB 2018, Paris, France, April 21-24, 2018, Proceedings, volume 10812, page 271. Springer, 2018.

[6] Zamin Iqbal, Mario Caccamo, Isaac Turner, Paul Flicek, and Gil McVean. De novo assembly and genotyping of variants using colored de bruijn graphs. Nature genetics, 44(2):226, 2012.

[7] Aleksey V Zimin, Kristian A Stevens, Marc W Crepeau, Daniela Puiu, Jill L Wegrzyn, James A Yorke, Charles H Langley, David B Neale, and Steven L Salzberg. An improved assembly of the loblolly pine mega-genome using long-read single-molecule sequencing. Gigascience, 6(1):1-4, 2017.

[8] Sergej Nowoshilow, Siegfried Schloissnig, Ji-Feng Fei, Andreas Dahl, Andy WC Pang, Martin Pippel, Sylke Winkler, Alex R Hastie, George Young, Juliana G Roscito, et al. The axolotl genome and the evolution of key tissue formation regulators. Nature, 554(7690):50, 2018. 
bioRxiv preprint doi: https://doi.org/10.1101/546309; this version posted April 28, 2020. The copyright holder for this preprint (which was

not certified by peer review) is the author/funder, who has granted bioRxiv a license to display the preprint in perpetuity. It is made available under aCC-BY-NC-ND 4.0 International license.

[9] Burton H Bloom. Space/time trade-offs in hash coding with allowable errors. Communications of the ACM, 13(7):422-426, 1970.

[10] Brad Solomon and Carl Kingsford. Fast search of thousands of short-read sequencing experiments. Nature biotechnology, 34(3):300, 2016.

[11] Rayan Chikhi and Guillaume Rizk. Space-efficient and exact de bruijn graph representation based on a bloom filter. Algorithms for Molecular Biology, 8(1):22, 2013.

[12] Shaun D Jackman, Benjamin P Vandervalk, Hamid Mohamadi, Justin Chu, Sarah Yeo, S Austin Hammond, Golnaz Jahesh, Hamza Khan, Lauren Coombe, Rene L Warren, et al. Abyss 2.0: resource-efficient assembly of large genomes using a bloom filter. Genome research, pages gr-214346, 2017.

[13] Paolo Ferragina and Giovanni Manzini. Indexing compressed text. Journal of the ACM (JACM), 52(4):552-581, 2005.

[14] Camille Marchet, Lolita Lecompte, Antoine Limasset, Lucie Bittner, and Pierre Peterlongo. A resource-frugal probabilistic dictionary and applications in bioinformatics. Discrete Applied Mathematics, 2018.

[15] Martin D Muggli, Alexander Bowe, Noelle R Noyes, Paul S Morley, Keith E Belk, Robert Raymond, Travis Gagie, Simon J Puglisi, and Christina Boucher. Succinct colored de bruijn graphs. Bioinformatics, 33(20):3181-3187, 2017.

[16] Avi Srivastava, Fatemeh Almodaresi, Hirak Sarkar, and Rob Patro. A space and time-efficient index for the compacted colored de Bruijn graph. Bioinformatics, 34(13):i169-i177, 062018.

[17] Antoine Limasset, Guillaume Rizk, Rayan Chikhi, and Pierre Peterlongo. Fast and scalable minimal perfect hashing for massive key sets. arXiv preprint arXiv:1702.03154, 2017.

[18] Fatemeh Almodaresi, Hirak Sarkar, and Rob Patro. A space and time-efficient index for the compacted colored de bruijn graph. bioRxiv, page 191874, 2017.

[19] Ilia Minkin, Son Pham, and Paul Medvedev. Twopaco: An efficient algorithm to build the compacted de bruijn graph from many complete genomes. Bioinformatics, 33(24):4024-4032, 2016.

[20] Rayan Chikhi, Antoine Limasset, and Paul Medvedev. Compacting de bruijn graphs from sequencing data quickly and in low memory. Bioinformatics, 32(12):i201-i208, 2016.

[21] Amatur Rahman and Paul Medvedev. Representation of k-mer sets using spectrum-preserving string sets. In RECOMB, 2020.

[22] Christina Boucher, Alex Bowe, Travis Gagie, Simon J Puglisi, and Kunihiko Sadakane. Variable-order de bruijn graphs. In 2015 Data Compression Conference, pages 383-392. IEEE, 2015.

[23] Rayan Chikhi, Antoine Limasset, Shaun Jackman, Jared T Simpson, and Paul Medvedev. On the representation of de bruijn graphs. Journal of Computational Biology, 22(5):336-352, 2015.

[24] Hongzhe Guo, Yilei Fu, Yan Gao, Junyi Li, Yadong Wang, and Bo Liu. degsm: memory scalable construction of large scale de bruijn graph. bioRxiv, page 388454, 2018.

[25] Prashant Pandey, Fatemeh Almodaresi, Michael A Bender, Michael Ferdman, Rob Johnson, and Rob Patro. Mantis: A fast, small, and exact large-scale sequence-search index. Cell Systems, 2018.

[26] Michael Roberts, Wayne Hayes, Brian R Hunt, Stephen M Mount, and James A Yorke. Reducing storage requirements for biological sequence comparison. Bioinformatics, 20(18):3363-3369, 2004.

[27] Yang Li et al. Mspkmercounter: a fast and memory efficient approach for k-mer counting. arXiv preprint arXiv:1505.06550, 2015.

[28] Sebastian Deorowicz, Marek Kokot, Szymon Grabowski, and Agnieszka Debudaj-Grabysz. Kmc 2: fast and resource-frugal k-mer counting. Bioinformatics, 31(10):1569-1576, 2015.

[29] Eric Karsenti, Silvia G Acinas, Peer Bork, Chris Bowler, Colomban De Vargas, Jeroen Raes, Matthew Sullivan, Detlev Arendt, Francesca Benzoni, Jean-Michel Claverie, et al. A holistic approach to marine eco-systems biology. PLoS biology, 9(10):e1001177, 2011.

[30] Prashant Pandey, Michael A Bender, Rob Johnson, and Rob Patro. Squeakr: an exact and approximate k-mer counting system. Bioinformatics, 34(4):568-575, 2017.

[31] Thomas C Conway and Andrew J Bromage. Succinct data structures for assembling large genomes. Bioinformatics, 27(4):479-486, 2011. 
[32] Dinghua Li, Chi-Man Liu, Ruibang Luo, Kunihiko Sadakane, and Tak-Wah Lam. Megahit: an ultra-fast single-node solution for large and complex metagenomics assembly via succinct de bruijn graph. Bioinformatics, 31(10):1674-1676, 2015.

[33] Prashant Pandey, Michael A Bender, Rob Johnson, and Rob Patro. Squeakr: an exact and approximate k-mer counting system. Bioinformatics, 34(4):568-575, 2018.

[34] Camille Marchet, Zamin Iqbal, Daniel Gautheret, Mikaël Salson, and Rayan Chikhi. Reindeer: efficient indexing of k-mer presence and abundance in sequencing datasets. In ISMB, 2020.

[35] Karel Břinda, Michael Baym, and Gregory Kucherov. Simplitigs as an efficient and scalable representation of de Bruijn graphs. bioRxiv, 2020.

[36] Guillaume Holley and Páll Melsted. Bifrost-highly parallel construction and indexing of colored and compacted de bruijn graphs. BioRxiv, page 695338, 2019. 